\title{
Mechanismus der Aktivitätsabnahme mischfunktioneller Oxygenasen in Lebermikrosomen urämischer Ratten
}

\author{
Von H. W. Leber \\ Aus den Medizinischen Kliniken und Polikliniken der Justus Liebig-Universität Gießen
}

(Eingegangen am 13. Juli/14. August 1972)

\begin{abstract}
An männlichen Ratten wurde durch subtotale Nephrektomie eine Urämie erzeugt. Am sechste.. postoperativen Tag wurden die Lebermikrosomen der urämischen Tiere und scheinnephrektomierter Kontrollen isoliert. Bei urämischen Ratten fand sich eine Abnahme der spezifischen Aktivität der Aminopyrindemethylicrung und des Cytochrom P-450-gehaltes auf 65 bzw. 68\% der Norm. Die Substratbindung hatte hinsichtlich Anilin auf 50\%, bezüglich Hexobarbital auf $63 \%$ abgenommen. Die Initialgeschwindigkeit der Cytochrom P-450-Reduktion durch NADPH verlief bei urämischen Tieren in Abwesenheit und Gegenwart von Substrat signifikant langsamer als bei Kontrolltieren. Die Aktivität der NADPH-Cytochrom c-Reduktasc wurde durch die Urämie nicht verändert. Kalorische Unterernährung allein hatte keinen Einfluß auf die untersuchten Parameter.
\end{abstract}

\section{Mechanism of uremia-induced decrease of the activity' of the mixed function oxidation system in rat liver microsomes}

On the sixth postoperative day, liver microsomes were isolated from subtotal nephrectomized and sham operated male rats. In liver microsomes from uremic rats, specific activity for the demethylation of aminopyrine decreased to $65 \%$, cytochrome P-450 content to $68 \%$, binding of Aniline to $50 \%$, and binding of Hexobarbital to $63 \%$ of the values measured with microsomes from controls. Initial velocity of the NADPH-dependent reduction of cytochrome P-450 was significant slower in microsomes from uremic rats, whether substrate was added or not. Specific activity of NADPH-cytochrome c-reductase was not altered in the uremic state. Caloric deficiency alone did not influence the measured parameters.

Frühere eigene Untersuchungen an subtotal nephrektomierten Ratten haben gezeigt, daß die Bedingungen der Urämie neben einer Abrnahme des absoluten und relativen Leberfeuchtgewichtes eine Verminderung des absoluten und auf $1 \mathrm{~g}$ Leber bezogenen relativen Gehaltes der Leber an mikrosomalem Protein bewirken. Diese Effekte sind nicht urämiespezifisch, denn entsprechende Veränderungen waren auch an scheinnephrektomierten, kalorisch unterernährten Kontrolltieren zu beobachten.

Im Gegensatz zur kalorischen Unterernährung verursachte die Urämie zusätzlich eine Abnahme der - auf $1 \mathrm{mg}$ Mikrosomenprotein bezogenen - spezifischen Aktivität mischfunktioneller Oxygenasen einschließlich des Gehalts an Cytochrom P-540. Die spezifische Altivität anderer mikrosomaler Enzyme - Glucose-6-Phosphatase (EC 3.1.3.9) und Glucuronyltransferase (EC 2.4.1.17) - sowie der Gehalt an Cytochrom $b_{5}$ wurden dagegen durch die Urämie nicht verändert $(1,2,3)$.

Vorbehandlung urämischer Ratten mit Phenobarbital (4) stimulierte die spezifische Aktivität der Aminopyrindemethylierung und den mikrosomalen Cytochrom P-450-gehalt wie bei scheinnephrektomierten Kontrolltieren: Die nach Phenobarbitalgabe gefundenen Werte lagen sowohl bei urämischen als auch bei scheinnephrektomierten Tieren signifikant höher als bei unbehandelten Kontrollen mit normaler Nierenfunktion.

Außerdem konnte gezeigt werden, daß die bei urämischen Ratten bestehende Abnahme des mikrosomalen
Cytochrom P-450-gehaltes auf eine Störung der Hämsynthese infolge eines Mangels an $\delta$-Aminolävulinsäure zurückzuführen ist, denn Vorbehandlung der Tiere mit $\delta$-Aminolävulinsäure normalisierte den erniedrigten Cytochrom P-450-gehalt (1). Die Normalisierung des Cytochrom P-450-gehaltes war nicht von einem entsprechenden Anstieg der spezifischen Aktivität mischfunktioneller Oxygenasen begleitet. Der erniedrigte mikrosomale Cytochrom P-450-gehalt ist somit allein nicht verantwortlich für die unter den Bedingungen der Urämie herabgesetzte spezifische Aktivität mischfunktioneller Oxygenasen.

Nach heutiger Auffassung setzt sich die mischfunktionelle Oxygenierung aus verschiedenen Teilreaktionen zusammen. Die wichtigsten Schritte der Reaktionskette sind die Oxidation von Cytochrom P-450, die Bindung von Substraten an oxidiertes Cytochrom P-450, die Redultion des Cytochrom P-450-Substratkomplexes durch NADPH, wobei das mikrosomale Flavoprotein NADPH-Cytochrom c-Reduktase (EC 1.6.99.3) eine wichtige Rolle spielt und möglicherweise ein noch nicht endgültig identifiziertes Nichthämeisenproteid beteiligt ist sowie der A.ktivierung und Anlagerung von molekularem Sauerstoff an den reduzierten Cytochrom P-450-Substratkomplex (5).

Ergebnisse von Ullrich (6) und Degkiwitz et al. (7) legen den Schluß nahe, daß Änderungen der spezifischen Aktivität mischfunktioneller Oxygenasen mit entsprechenden Änderungen der Kapazität zur Substratbindung an Cytochrom P-450 einhergehen. Aus den Untersuchungen von Grgon et al. (8) sowie Diehr 
et al. (9) wurde geschlossen, daß die initiale Reduktionsgeschwindigkeit des Cytochrom P-450-Substratkomplexes die mischfunktionelle Oxygenierung von Typ I-Substraten limitiert.

$\mathrm{Da}$ die in Lebermikrosomen urämischer Ratten beobachtete Abnahme der spezifischen Aktivität mischfunktioneller Oxygenasen nicht nur durch die gleichzeitige Verminderung des mikrosomalen Cytochrom P-450-gehaltes verursacht wird, sollte geklärt werden, an welchen Teilschritten der mischfunktionellen Oxygenierung die Bedingungen der Urämie zusätzlich Einflüsse entfalten.

$\mathrm{Zu}$ diesem $\mathrm{Zweck}$ wurden in Lebermikrosomen urämischer Ratten und scheinnephrektomierter Kontrolltiere folgende Parameter gemessen:

1. Aktivität der NADPH-Cytochrom c-Reduktase.

2. Reduktion des Cytochrom P-450 durch NADPH in Abwesenheit und nach Zugabe von Substrat.

3. Bindung von Hexobarbital und Anilin an Cytochrom P-450.

4. Aktivität der N-Demethylierung von Aminopyrin.

5. Cytochrom P-450-gehalt.

\section{Methodik}

\section{Versucbs- und Kontrolltiere}

Die Versuche wurden an 50-70 Tage alten männlichen SIVRatten der $\mathrm{Fa}$. Ivanovas (Kisslegg/Allgäu) mit einem Ausgangsgewicht von ungefähr $250 \mathrm{~g}$ durchgefühtt (1).

Die Urämie wrurde durch subtotale Nephrektomie erzeugt, wobei $12-15 \%$ des Parenchyms beider Nieren zusammen unter sorgfältiger Schonung beider Nebennieren erhalten blieben.

Als Kontrollen dienten scheinnephrektomierte Ratten, die Futter und Wasser ad libitum erhielten sowie scheinnephrektomierte Tiere, die so gefüttert wurden, daß sie in der gleichen Zeit denselben Gewichtsverlust erlitten wie subtotal nephrektomierte Tiere (sogenannte kalorisch unterernährte oder Hungertiere). Einzelheiten der subtotalen Nephrektomie, der Scheinnephrektomie sowie der Tierhaltung sind in einer früheren Arbeit angegeben (1).

\section{Präparation der Lebermikrosomen}

6 Tage nach subtotaler Nephrektomie bzw. Scheinnephrektomie wurden die Tiere in Äthernarkose durch Entbluten über die Aa. carotides getötet.

Entsprechend früheren Angaben (10) wurde die Leber in 0,1 mol/1 Tris- $\mathrm{HCl}$ Puffer $\mathrm{pH} 7,4$ mit $0,25 \mathrm{~mol} / \mathrm{l}$ Saccharosegehalt unter Kühlung mit Eis homogenisiert ( $5 \mathrm{ml}$ Puffer/g Leber).

Anschließend wurde die Mikrosomenfraktion in Anlehnung an SCHNEIDER (11) wie in einer eigenen früheren Arbeit angegeben (10) durch Differentialzentrifugation isoliert.

Um Hämoglobinverunreinigungen zu beseitigen, wurden die Mikrosomen mit isotoner Kaliumchloridlösung gewaschen und anschließend in 0,1 mol/l Tris- $\mathrm{HCl}$ Puffer $\mathrm{pH} 7,4$ aufgenommen (10).

\section{Proteinbestimmung}

Der Proteingehalt der Mikrosomensuspension wurde mit dem Biuretreagenz nach BeISENHerz et al. (12) bestimmt. Die bei dieser Methode störende unspezifische Trübung wurde durch Zusatz von $\mathrm{KCN}$ nach Bode et al. (13) erfaßt.

\section{Bestimmung der Aktivität der N-Demetbylierung von Aminopyrin}

Einzelheiten des Ansatzes wurden in einer früheren Arbeit veröffentlicht (10). Der bei der Umsetzung von Aminopyrin entstehende Formaldehyd wurde nach NASH (14) gemessen. Ein Leerwert enthielt anstelle von Aminopyrin ein entsprechendes Volumen an Puffer.

\section{Bestimmung des mikrosomalen Cytochrom P-540-gebaltes}

Der Cytochrom P-450-gehalt wurde nach OMURA und SaTo (15) entsprechend früheren Angaben (10) gemessen. Zur Berechnung wurde der Extinktionskoeffizient E450-490nm $=91 \mathrm{~cm}^{2} / \mathrm{mmol}$ (16) zugrunde gelegt.

\section{Messung der NADPH-Cytocbrom P-450-Reduktion}

Die Reduktion wurde entsprechend den Angaben von GigoN et al. (17) bei $30^{\circ} \mathrm{C}$ an der Zunahme der Extinktionsdifferenz zwischen $450 \mathrm{~nm}$ und $470 \mathrm{~nm}$ in einem Aminco-Chance-DualWavelength Split Beam Recording Spectrophotometer (Silverspring, Maryland, USA) gemessen. Der Ansatz enthielt in 2,5 ml

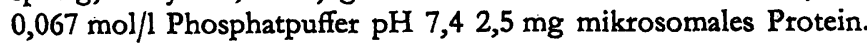
Zur Untersuchung des Einflusses von Typ I-Substraten auf die Initialgeschwindigkeit der Cytochrom P-450-Reduktion wurde einzelnen Ansätzen Aminopyrin in einer Endkonzentration von $5 \mathrm{mmol} / \mathrm{l}$ zugesetzt. Jedem Ansatz wurden außerdem $25 \mu \mathrm{mol}$ Glucose, $0,2 \mathrm{mg}$ Glucoseoxidase (EC 1.1.3.4) und $130 \mathrm{mU}$ Katalase (EC 1.11.1.6) (9) zugesetzt, um den Sauerstoff vollständig aus der Küvette zu entfernen. Jeder Ansatz wurde 3 min mit $\mathrm{O}_{2}$-freiem $\mathrm{CO}$ begast, nach vierminütigem Temperaturausgleịch im beheizten Küvettenhalter wurde. die Cytochrom P-540Reduktion durch schnelle Zugabe von $50 \mu \mathrm{l}$ eines NADPHregenerierenden Systems (Endkonzentrationen: 0,4 mmol/1 NADP, $4 \mathrm{mmol} / 1$ Isocitrat, $3 \mathrm{mmol} / 1 \mathrm{MgCl}_{2}$ und $5 \mu \mathrm{l}$ Isocitratdehydrogenase, (EC 1.1.1.41) $20 \mathrm{mg} / 2 \mathrm{ml}$ ) in der luftdicht verschlossenen Küvette gestartet. Die Cytochrom P-450-Reduktion wurde während der ersten $7 \mathrm{~min}$ kontinuierlich registriert, die dann gemesssene Extinktionsdifferenz wurde als $100 \%$ NADPHreduzierbares ( $=$ oxidiertes) Cytochrom P-450 festgesetzt. Anschließend wurde durch Zugabe einiger Körnchen Natriumdithionit der Dithionit-reduzierbare Anteil gemessen. In Úbereinstimmung mit DreHr (9) wurde gefunden, daß in allen Kollektiven durch NADPH etwa $95 \%$ des Dithionit-reduzierbaren Anteils reduziert werden.

\section{Bestimmung der Aletivität der NADPH-Cjtocbrom i-Reduktase}

Die Reduktion von Cytochrom c durch NADPH wurde bei $25^{\circ} \mathrm{C}$ entsprechend den Angaben von Wrutiams et al. (18) in einem Aminco-Chance-Dual Wavelength Split Beam Recording Spectrophotometer (Springfield, Maryland, USA) an der Zunahme der Extinktionsdifferenz zwischen 550 und $542 \mathrm{~nm}$ gemessen. Die Ansätze enthielten in $2 \mathrm{ml} 0,067 \mathrm{~mol} / 1$ Phosphatpuffer $\mathrm{pH} 7,70,08 \mathrm{mmol} / 1$ Cytochrom c, $1,0 \mathrm{mmol} / 1 \mathrm{NADPH}$, $1,0 \mathrm{mmol} / \mathrm{l} \mathrm{KCN}$ und pro $\mathrm{ml}$ Ansatz $5-10 \mu \mathrm{g}$ mikrosomales Protein.

Zur Berechnung der Aktivität der NADPH-Cytochrom c-Reduktase wurde ein Extinktionskoeffizient von $E_{550-542 ~ n m}=21 \mathrm{~cm}^{2} /$ mmol (19) zugrunde gelegt.

\section{Messung der Substratbindung von Hexobarbital und Anilin}

Die Differenzspektren wurden mit einem Cary Modell 14, Scattered Transmission Accessory, bei Raumtemperatur registriert. Die Ansätze enthielten in $8 \mathrm{ml} 0,1 \mathrm{~mol} / 1$ Trispuffer $\mathrm{pH} 7,416 \mathrm{mg}$ Mikrosomenprotein. In die Meß- und Vergleichsküvette wurden je 2,8 ml dieser Mikrosomensuspension pipettiert. Nach der Registrierung des Basisdifferenzspektrums (sog. Nullinie) wurde der Meßküvette $0,2 \mathrm{ml}$ Substratlösung, der Vergleichsküvette $0,2 \mathrm{ml}$ dest. Wasser zugesetzt. Anschließend wurde erneut das Differenzspektrum zwischen 350 und $450 \mathrm{~nm}$ aufgenommen. Die Endkonzentrationen an Substrat betrugen sowohl für Anilin als auch für Hexobarbital-Na $5 \mathrm{mmol} / 1$ (gelöst in dest. Wasser). Als Ausdruck der Bindung von Anilin wurde $\mathrm{E}_{430-390} \times \mathrm{mg}$ Protein ${ }^{-1}$ $\times \mathrm{cm}^{-1}$, als $\mathrm{Maß}$ für die Bindung von Hexobarbital $\mathrm{E}_{388-420} \times \mathrm{mg}$ Protein ${ }^{-1} \times \mathrm{cm}^{-1}$ bestimmt (20).

\section{Spezifische Enzymaktivitäten}

Die spezifischen Aktivitäten der gemessenen Enzyme wurden in nmol Produkt $\times \mathrm{mg}$ Protein ${ }^{-1} \times \mathrm{min}^{-1}$ angegeben.

Die Reduktion des Cytochrom P-450-Substratkomplexes weist eine komplizierte Kinetik auf (9), bei der zwei Phasen interferieren: eine schnelle Phase verläuft entsprechend einer Reaktion 


\title{
Laborgeräte aus PTFE
}

\author{
(Hostaflon(R) Teflon(R) \\ Autoklaven und Filtrationszellen \\ Membranen und Filterplatten
}
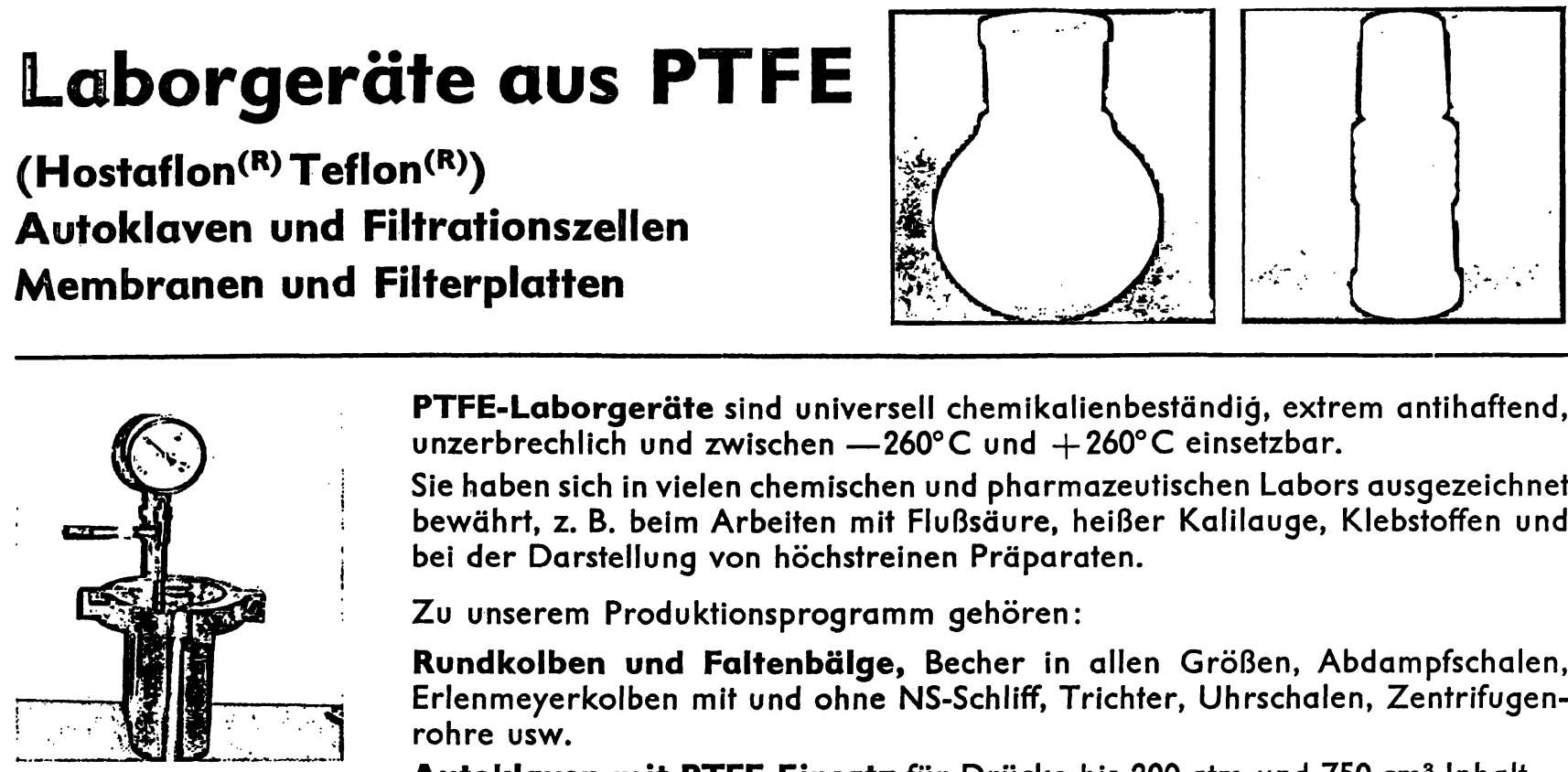

PTFE-Laborgeräte sind universell chemikalienbeständig, extrem antihaftend, unzerbrechlich und zwischen $-260^{\circ} \mathrm{C}$ und $+260^{\circ} \mathrm{C}$ einsetzbar.

Sie haben sich in vielen chemischen und pharmazeutischen Labors ausgezeichnet bewährt, z. B. beim Arbeiten mit Flußsäure, heißer Kalilauge, Klebstoffen und bei der Darstellung von höchstreinen Präparaten.

Zu unserem Produktionsprogramm gehören:

Rundkolben und Faltenbälge, Becher in allen Größen, Abdampfschalen, Erlenmeyerkolben mit und ohne NS-Schliff, Trichter, Uhrschalen, Zentrifugenrohre usw.

Autoklaven mit PTFE-Einsatz für Drücke bis $200 \mathrm{~atm}$ und $750 \mathrm{~cm}^{3}$ Inhalt.

Filtrationszellen aus Kunststoff oder Edelstahl für hydrostatische Drücke bis zu $150 \mathrm{~atm}$.

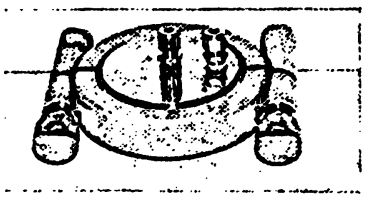

Membranen und Filterplatten mit verschiedenen Porengrößen zwischen 0,05 und $50 \mu$ zur Reinigung, Trennung und Konzentrierung von Mischungen und makromolekularen Lösungen.

Nach Ihren speziellen Anforderungen stellen wir Sonderapparate her.

\section{Forschungsinstitut Berghof GmbH, 74 Tübingen-Lustnau Tel.07122-5035}




Dosier- und Pipettiereinheiten

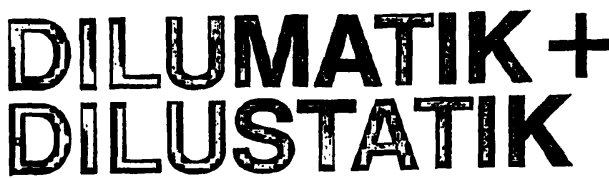

Grundbausteine für Automatisierungsaufgaben im Labor, austauschbar z. B. zum Verdünnungsautomaten bis zur vollständigen Analysenstraße.

DILUSTATIK: "stapelbar «, d. h. gemeinsamer Betrieb beliebig vieler Geräte von einer Steuereinheit aus.

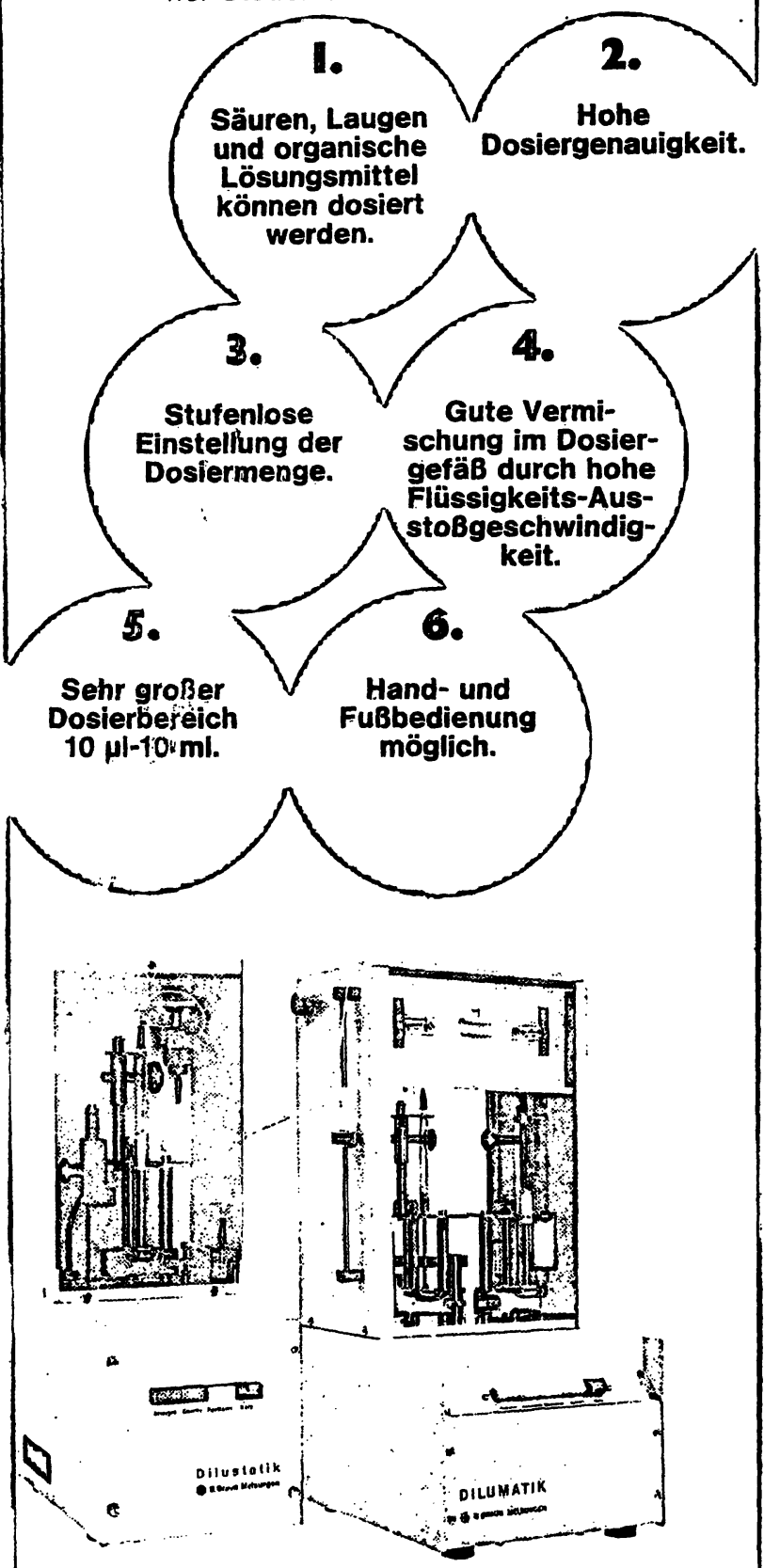

.

\section{B. Braun Melsungen Aktiengesellschat} Medizin-u.Labortechnik 3508 Melsungen, Postfach 110 und 120
Walter de Gruyter Berlin. New York

Neuerscheinung

\section{Fuchs - Freiwald}

\section{Allgemeine und Anorganische Chemie}

Einführung in die Grundlagen für Mediziner, Naturwissenschaftler und Chemie-Nebenfächler

Von Prof. Dr. JOACHIM FUCHS und Dipl.-Chem. WILFRIED FREIWALD, Institut für Anorganische Chemie der Freien Universität Berlin

Groß-Oktav. VI, 174 Seiten. 1972

Plastik flexibel DM 12,80

ISBN 3110042436

Der "Lerntext" soll in kürzester, aber dennoch verständlicher Form die wichtigsten Grundlagen der anorganischen und allgemeinen Chemie darstellen. Dabei ist der "Lernstoff" auf ein Minimum beschränkt, das Hauptgewicht wird auf ein Vermitteln allgemeiner Gesetzmäßigkeiten und Zusammenhänge gelegt.

Nach Kapiteln über Atombau und Periodensystem wird die Chemie der Elemente, geordnet nach den Gruppen des Periodensystems, besprochen, wobei an prägnanten Beispielen Gebiete aus der allgemeinen und physikalischen Chemie (Reaktionskinetik, Thermodynamik, Elektrochemie) in einfacher ${ }^{\mathrm{F}}$.orm behandelt werden. Der Aufbau ist so gewählt, daß die Eigenschaften und Reaktionen der Stoffe mit Hilfe der jeweils bereits erklärten theoretischen Grundlagen anschaulich verständlich sind. Schon nach einem einmaligen intensiven Durcharbeiten des Stoffes wird ein hoher Lerneffekt erzielt.

Das Buch ist speziell für Studenten mit Chemie als Nebenfach (Mediziner, Biologen, Physiker u. a.) gedacht. Es sollte aber auch für Studenten der Chemie als Hauptfach und für Chemotechniker an

Ingenieurakademien und Fachhochschulen in den Anfangssemestern von Nutzen sein, um zu erkennen, welche Gebiete der Chemie, die in ausführlichen und umfangreichen Lehrbüchern behandelt werden, von besonderer Wichtigkeit sind.

For USA and Canada:

Please send all orders to Walter de Gruytar Inc.

162 Fifth Avenue, New York N.Y. 10010 Tel. (212) 255-0808 
1. Ordnung, die in eine langsame Phase übergeht. Die Reaktionsgeschwindigkeit ist initial demnach vom Gehalt der Mikrosomen an oxidiertem Cytochrom P-450 abhängig. Um zu klären, ob statistisch gesicherte Unterschiede bezüglich der Initialgeschwindigkeit der Cytochrom P-450-Reduktion in An- und Abwesenheit von Aminopyrin zwischen urämischen Ratten und Kontrolltieren bestehen, wurde eine Varianzanalyse von 10 Meßpunkten während der ersten $20 \mathrm{~s}$ des Reaktionsablaufes durchgeführt. Anschließend wurden die bei den einzelnen Kollektiven erhaltenen Kurvenmittelwrerte miteinander verglichen.

\section{Bestivmung der Serumkonzentrationen von Harnstoff und Kreatinin}

Die Messungen wurden nach Routineverfahren im Autoanalyzer des Klin.-Chem. Institutes der Universirät Gießen durchgeführt.

\section{Statistiscbe Auswertung}

Für die statistischen Berechnungen wurde - abgesehen von der Auswertung der NADPH-Cytochrom P-450-Reduktion - der $\chi$-Test benutzt (21). In den Tabellen ist außer dem Mittelwert die Standardabweichung als Ausdruck der Streuung angegeben. Signifikante Differenz = Irrtumswahrscheinlichkeit bei einseitiger Anwendung des Testes $=1 \%$ und darunter.

\section{Ergebnisse}

In Tabelle 1 sind die Serumkonzentrationen von Harnstoff und Kreatinin bei subtotal und scheinnephrektomierten Ratten am 6 . postoperativen Tage angegeben. Entsprechend früher mitgeteilten Befunden $(1,2)$ bewirkte die subtotale Nephrektomie einen signifikanten Anstieg der Serumkonzentrationen von Harnstoff und

Tab. 1

Harnstoff- und Kreatininkonzentrationen im Serum subtotal nephrektomierter Versuchs- und scheinnephrektomierter Kontrolltiere am 6. postoperativen Tag

Signifikante Unterschiede $(p<0,01)$ : $\times$ verglichen mit 0

\begin{tabular}{lcc}
\hline & $\begin{array}{c}\text { Harnstoff } \\
(\mathrm{mmol} / \mathrm{l}) \\
\overline{\mathbf{x}} \pm \mathrm{s}\end{array}$ & $\begin{array}{c}\text { Kreatinin } \\
(\mathrm{mmol} / \mathrm{l}) \\
\overline{\mathbf{x}} \pm \mathrm{s}\end{array}$ \\
\hline $\begin{array}{c}\text { Normal ernährte Kontrolltiere } \\
\mathbf{n}=25\end{array}$ & $\times_{8,3} \pm 0,5$ & $\times_{0,04} \pm 0,008$ \\
$\begin{array}{c}\text { Kalorisch unterernāhrte Tiere } \\
\mathbf{n}=25\end{array}$ & $\times 8,7 \pm 0,8$ & $\times_{0,05} \pm 0,015$ \\
$\begin{array}{c}\text { Subtotal nephrektomierte Tiere } \\
\mathrm{n}=25\end{array}$ & ${ }^{\circ} 61,6 \pm 13,4$ & ${ }^{\circ} 0,42 \pm 0,11$ \\
\hline
\end{tabular}

Kreatinin. Die bei kalorisch unterernährten Tieren gefundenen Werte unterschieden sich nicht von den bei normal ernährten scheinnephrektomierten gemessenen. Hinsichtlich des Verhaltens von Körpergewicht, der Lebergewichte und der Ausbeute an mikrosomalem Protein wird auf eine frühere Arbeit verwiesen (1).

Wie aus Tabelle 2 hervorgeht, war $6 \mathrm{~d}$ nach subtotaler Nephrektomie neben der bereits früher beschriebenen Abnahme des spezifischen Cytochrom P-450-gehaltes und der spezifischen Aktivität der Aminopyrindemethylierung $(1,2)$ eine signifikante Verminderung der Substratbindung zu beobachten.

Gegenüber scheinnephrektomierten Kontrollen wurde bei urämischen Ratten ein Absinken der Extinktionsdifferenz nach Zusatz von Hexobarbital auf $62,5 \%$, nach Zusatz von Anilin auf 50\% gefunden. Bei kalorisch unterernährten Tieren, die in der postoperativen Phase den gleichen Gewichtsverlust erlitten hatten wie subtotal nephrektomierte Ratten, traten gegenüber normal ernährten Tieren keine signifikanten Unterschiede auf. Substratbindung von Hexobarbital, spezifische Aktivität der Aminopyrindemethylierung und spezifischer Cytochrom P-450-gehalt nahmen bei urämischen Tieren im Vergleich zu Kontrolltieren in demselben Verhältnis ab.

Tabelle 2 gibt auch die bei urämischen Ratten und Kontrolltieren gemessenen spezifischen Aktivitäten der NADPH-Cytochrom c-Reduktase wieder. Es ist ersichtlich, daß kalorische Unterernährung und Urämie keine Aktivitätsänderung dieses Enzyms bewirken. Abbildung 1 gibt die Initialgeschwindigkeit der NADPHCytochrom-P-450-Reduktion in An- und Abwesenheit von $5 \mathrm{mmol} / \mathrm{l}$ Aminopyrin wieder. Es sind die bei den einzelnen Kollektiven zu jedem Zeitpunkt ermittelten Kurvenmittelwerte in Prozent des Ausgangswertes angegeben $(100 \%$ oxidiertes Cytochrom $\mathrm{P}-450=$ $E_{450-470 \mathrm{~nm}} 7 \mathrm{~min}$ nach Zugabe des NADPH-regenerierenden Systems). Die Daten der Varianzanalyse sind in Tabelle 3 wiedergegeben.

Aus Abbildung 1 geht hervor, daß bei beiden Kollektiven Zusatz von $5 \mathrm{mmol} / \mathrm{l}$ Aminopyrin eine signifikante Zunahme der Initialgeschwindigkeit der Cyto-

Tab. 2

Spezifische Aktivitäten der Aminopyrindemethylierung und der NADPH-Cytochrom c-Reduktase, Cytochrom P-450-gehalt sowie Bindung von Anilin und Hexobarbital in Rattenlebermikrosomen $6 \mathrm{~d}$ nach subtotaler Nephrektomie bzw. Scheinnephrektomie. Bei der gewählten Konzentration von Hexobarbital und Anilin ( $5 \mathrm{mmol} / \mathrm{l}$ ) lag Substratsättigung vor, eine Eigenhemmung der Substratbindung war nicht festzustellen Signifikante Unterschiede $(p<0,01): *$ gegen +

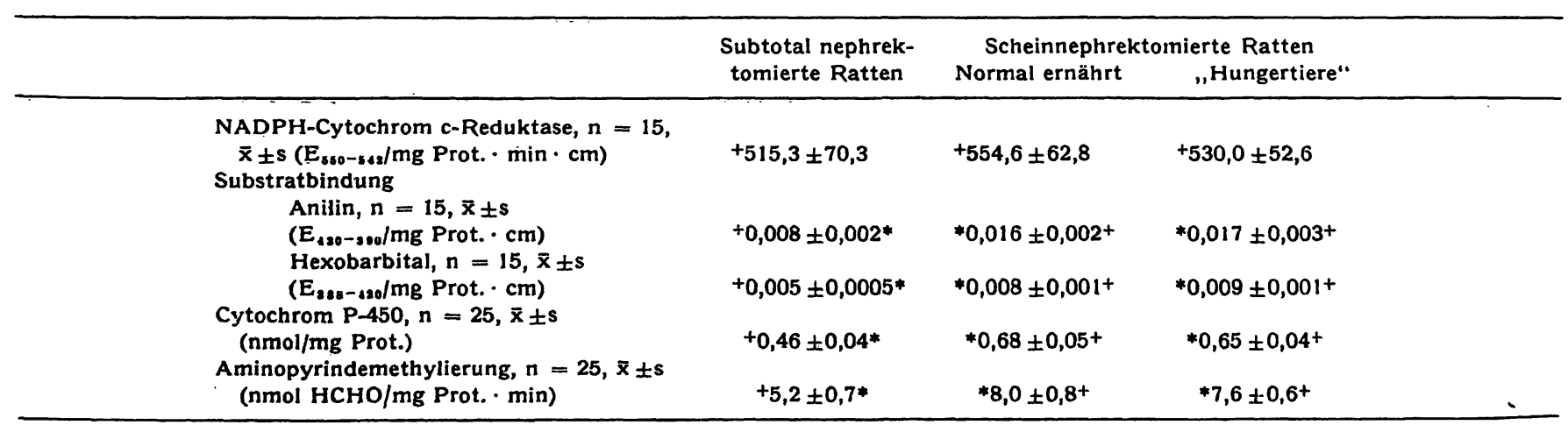

Z. Klin. Chem. Klin. Biochem. / 10. Jahrg. 1972/ Heft 12 
Tab. 3

Daten der Varianzanalyse zu Abbildung 1

Einflıßgrößen: A: Zeit, B: Tierkollektive, C: Urämie, D: Substrat. Signifikante Unterschiede $(p<0,01)$ sind mit $\times$ gekennzeichnet

\begin{tabular}{|c|c|c|c|c|c|}
\hline Einflußgröße & $\begin{array}{l}\text { Summe der } \\
\text { Abweichungs- } \\
\text { quadrate }\end{array}$ & $\begin{array}{l}\text { Freiheits- } \\
\text { grade }\end{array}$ & $\begin{array}{c}\text { Mittlere Ab- } \\
\text { weichungs- } \\
\text { quadrate }\end{array}$ & $\begin{array}{l}\text { Zufällige } \\
\text { Variable der } \\
\text { F-Verteilung }\end{array}$ & Signifikanz \\
\hline D & 40042,01 & 1 & 40042,01 & 133,69 & $x$ \\
\hline$C(D)$ & 17350,70 & 2 & 8687,85 & 29,01 & $x$ \\
\hline$B(C(D))$ & 10782,39 & 36 & 299,51 & - & .1 \\
\hline A & 37970,11 & 9 & 4218,90 & 394,79 & \\
\hline$A(D)$ & 6438,75 & 9 & 715,42 & 66,95 & $x$ \\
\hline$A(C(D))$ & 2074,13 & 18 & 115,23 & 10,78 & $x$ \\
\hline Rest & 3462,43 & 324 & 10,69 & - & \\
\hline
\end{tabular}

Abb. 1

Cytochrom P-450-Reduktion durch NADPH bei urämischen Ratten (a) und Kontrolltieren (b) in Abwesenheit von Substrat (O) und nach Zugabe von Aminopyrin $(O)$ in einer Endkonzentration von $5 \mathrm{mmol} / \mathrm{l}$

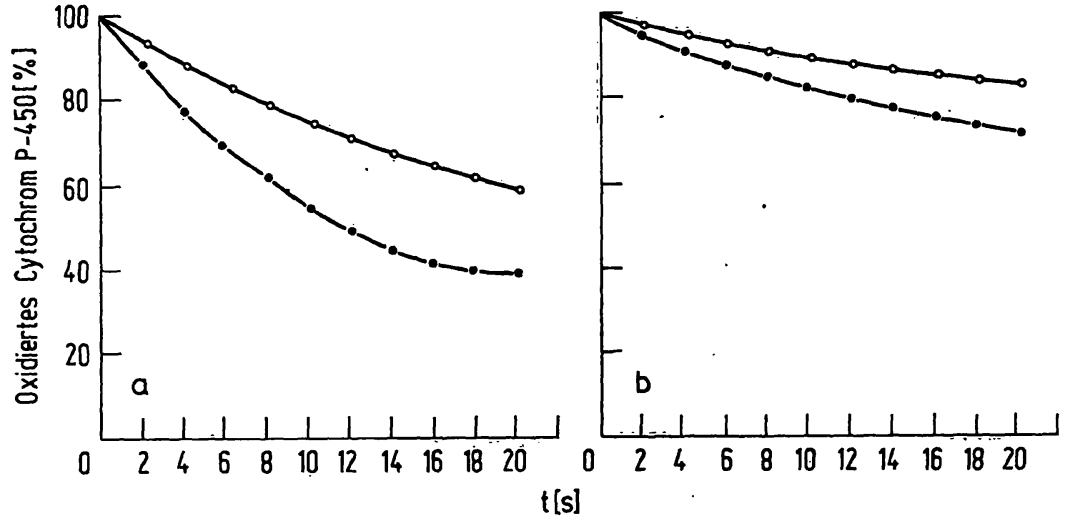

chrom P-450-Reduktion bewirkte. Sowohl in Gegenwart als auch in Abwesenheit von Aminopyrin verlief die Reaktion bei urämischen Tieren signifikant langsamer als bei scheinnephrektomierten Kontrollen. Aminopyrinzusatz verursachte bei Kontrolltieren eine stärkere Beschleunigung der initialen Cytochrom P450-Reduktion als bei urämischen Ratten. Kalorisch unterernährte Kontrolltiere verhielten sich hinsichtlich der Cytochrom P-450-Reduktion in Gegenwart und Abwesenheit von Substrat wie normal ernährte Kontrolltiere (unveröffentlichte Befunde).

\section{Diskussion}

Eigene frühere Untersuchungen haben gezeigt, daß Lebermikrosomen urämischer Ratten hinsichtlich der mischfunktionellen Oxygenierung eine verminderte Aktivität aufweisen (1-4). Das Zustandekommen dieser urämiebedingten Störung der mischfunktionellen Oxygenierung ist aufgrund der jetzt vorgelegten Befunde erklärbar.

Die Aufrechterhaltung der normalen spezifischen Aktivität mischfunktioneller Oxygenasen setzt das ungestörte Ineinandergreifen verschiedener Glieder einer Reaktionskette voraus (5). Es konnte gezeigt werden, da $B$ unter. den Bedingungen der Urämie einzelne Komponenten dieser Reaktionskette beeinträchtigt werden.

Aufgrund ihrer Bedeutung für die Geschwindigkeit der Oxygenierung von Typ I-Substraten ist die beim Vorliegen einer Urämie beobachtete Verlangsamung der Reduktion des Cytochrom P-450-Substratkomplexes hervorzuheben.
Die Reduktion des Cytochrom P-450-Substratkomplexes wird beeinflußt von

1. der spezifischen Akțivität der NADPH-Cytochrom c-Reduktase Elektŗonen direkt oder unter Vermittlung eines noch hypothetischen Carriers auf Cytochrom P-450 zu übertragen,

2. durch die Menge des pro mg Mikrosomenprotein zur Verfügung stehenden Cytochrom P-450 und

3. seiner Fähigkeit Substrate zu binden (5).

In unseren Untersuchungen wurde die spezifische Aktivität der NADPH-Cytochrom c-Reduktase in Lebermikrosomen urämischer Rattẹn gegenüber Kontrolltieren nicht verändert gefunden, es war aber eine statistisch gesicherte Beeinträchtigung der. Substratbindung an Cytochrom P-450 nachweisbar.

Daher ist anzunehmen, daß neben der Verminderung des spezifischen Cytochrom P-450-gehaltes die gleichzeitig bestehende Einschränkung der Substratbindungskapazität für die bei urämișchen Ratten beobachtete verlangsamte Reduktion des Cytochrom P=450-Substratkomplexes verantwortlich ist, wenn man bis jetzt nicht faßbare Aktivitätsänderungen des hypothetischen Carriers außer acht läßt.

Die nach Zusatz von Hexobarbital und Anilin mit Lebermikrosomen urämischer Ratten gefundene Änderung des Differenzspektrum war signifikant kleiner als bei scheinnephrektomierten Kontrolltieren.

ULLRICH (6) konnte mit Lebermikrosomen normaler Ratten hinsichtlich der Bindung von Cyclohexan nachweisen, daß nur ein Teil des im CO-Differenzspektrum gemessenen Cytochrom P-450 Substrat bindet. So wurde z. B. gezeigt, daß bei unbehandelten Kontroll- 
tieren $12 \%$, nach Phenobarbitalbehandlung aber 32\% des vorhandenen Cytochrom P-450 an der Bildung des Enzym-Substratkomplexes beteiligt ist.

Bezogen auf $1 \mathrm{mg}$ Mikrosomenprotein war bei urämischen Ratten im Vergleich zu Kontrolltieren die Bindung von Hexobarbital (Typ I) in dem gleichen Maße abgesunken wie der Cytochrom P-450-gehalt. Demzufolge war die Bindung von Hexobarbital pro nmol Cytochrom P-450 bei allen Kollektiven gleich. Daraus ist zu schließen, daß die bei urämischen Ratten - bezogen auf $1 \mathrm{mg}$ Mikrosomenprotein - verminderte Bindung von Hexobarbital alleine Folge der Abnahme des spezifischen Cytochrom P-450-gehaltes ist. Offenbar ist bei urämischen Ratten und Kontrolltieren der gleiche Anteil des im CO-Differenzspektrum gemessenen Cytochrom P-450 an der Bindung von Typ ISubstraten beteiligt.

Hinsichtlich der Bindung von Anilin (Typ II) war in Lebermikrosomen urämischer Ratten auch pro nmol Cytochrom P-450 eine Abnahme der für die Bindung typischen Extinktionsdifferenz festzustellen. Dies erlaubt den Schluß, daß unter den Bedingungen der Urämie ein kleinerer Anteil des vorhandenen Cytochrom P-450 Typ II-Substrate bindet als bei scheinnephrektomierten Kontrollen.

$\mathrm{Ob}$ die früher (1) am Beispiel der Acetanilidhydroxylierung beschriebene Abnahme der spezifischen Aktivität des Abbaues von Typ II-Substraten auf die verminderte Bindung an Cytochrom P-450 zurückgeht, kann nicht entschieden werden, solange nicht bekannt ist, welcher Schritt die Hydroxylierung von Typ IISubstraten limitiert.

Bezüglich der̃ spezifischen Aktivität der NADPHCytochrom c-Reduktase ist erwähnenswert, daß auch bei längerer Dauer der Urämie (bis zu 45 Tagen nach der subtotalen Nephrektomie) keine Unterschiede zu scheinnephrektomierten Kontrolltieren auftraten (eigene unveröffentlichte Befunde).
Die Abnahme der spezifischen Aktivität der mischfunktionellen Oxygenierung von Typ I-Substraten in Lebermikrosomen urämischer Ratten ist aufgrund unserer bisherigen Untersuchungen zurückzuführen auf:

1. die Verminderung des spezifischen Cytochrom P-450-gehaltes, ausgelöst durch einen Mangel an $\delta$ Aminolävulinsäure (1).

2. die damit einhergehende eingeschränkte Kapazität zur Substratbindung und

3. die Herabsetzung der Initialgeschwindigkeit der Reduktion des Cytochrom P-450-Substratkomplexes durch NADPH.

Die Messung der Schlafzeit urämischer Ratten nach Hexobarbital- oder Pentobarbitalinjektion hat ergeben, $\mathrm{d} a ß$ beim Vorliegen einer Urämie in vivo Typ I-Substrate wesentlich langsamer abgebaut werden als dies aufgrund der Abnahme des Gehaltes der Leber an Mikrosomenprotein und der in vitro beobachteten Verminderung der spezifischen Aktivität mischfunktioneller Oxygenasen zu vermuten war (in Vorbereitung). Es muß daher geprüft werden, durch welche zusätzlichen Faktoren der oxidative Axzneimittelstoffwechsel beim Vorliegen einer Urämie in vivo weiter beeinträchtigt wird.

\section{Danksagung}

Mit Unterstützung der Deutschen Forschungsgemeinschaft.

Die Messungen wurden teilweise mit Geräten des Biochemischen Institutes der Justus Liebig-Universität Gießen durchgeführt. Hierfür sei Herrn Prof. Dr. HJ. Staudinger auch an dieser Stelle noch einmal herzlich gedankt.

Frau E. M. Berg danke ich für die geleistete technische Mitarbeit.

Herrn Dipl.-MATH. HöBEL, Institut für Dokumentation und Statistik der Justus Liebig-Universität Gießen, danke ich für wertvolle Ratschläge sowie für die durchgeführten Berechnungen.

\section{Literatur}

1. LEBER, H.'W. \& SchürTrRRLE, G. (1972), Kidney International, im Druck. - 2. Leeber, H. W., Streitzig, P., KaYYSer, M. \& SChÜTtERLE, G. (1971), Klin. Wochenschr. 49, 944-945. 3. Leber, H. W., Streitzig, P., Kayser, M. \& Schürterle, G. (1971), Internat. Symposion on Uremia, Freiburg, im Druck. 4. Leber, H. W., Streitzig, P., Kayser, M. \& Schütterle, G. (1971), Tagg. der Dtsch. Ges. f. Nephrologie, Aachen, im Druck. 5. Gillette, J. R. (1971), Ann. N. Y. Acad. Sci. 179, 43-66. 6. UlllRich, V. (1969), Hoppe-Seyler's Z. Physiol. Chem. 350 , 357-365. - 7. Degkwitz, E., Ullrich, V. \& Staudinger, HJ. (1969), Hoppe-Seyler's Z Z. Physiol. Chem. 350, 547-553. - 8. Gigon, P. L., Gram, T. E. \& Grletette, J. R. (1969), Molec. Pharmacol. 5, 109-122. - 9. Diehi, H., Schüdelis, J. \& Ullrich, V. (1970), Hoppe-Seyler's Z. Physiol. Chem. 351, 1359-1371. - 10. Leber, H. W., Degkwitz, E. \& Staudinger, HJ. (1969), Hoppe-Seyler's Z. Physiol. Chem. 350, 439-445. 11. SChneider, W. C. (1948), J. Biol. Chem. 176, 259-266. -
12. Beisenherz, G., Boltze, H. J., Bücher, Th., Czok, R., Garbade, K. H., Meyer-Arendt, E. \& Pfleiderer, G. (1953), Z. Naturforschg. B, 8, 555-577. - 13. Bode, Ch., Goebell, H. \& StähleR, E. (1968), diese Z. 6, 418-422. - 14. NASh, T. (1953), Biochem. J. 55, 416-421. - 17. OMURA, T. \& SATO, R. (1964), J. Biol. Chem. 239, 2370-2378. - 16. OMURA, T. \& Sato, R. (1964), J. Biol. Chem. 239, 2379-2385. - 17. Gigon, P. L., Gram, T. E. \& GilletTe, J. R. (1968), Biochem. Biophys. Res. Commun. 31, 558-562. - 18. Williams, C. \& Kamin, H. (1962), J. Biol. Chem. 237, 587-595. - 19. MASSEY, V. (1959), Biochim. Biophys. Acta 34, 255-256. - 20. Remarer, H., Schenkman, J., Estabrook, R. W., Sasame, H., Gillette, J. R., Narastmhulu, S., Cooper, D. Y. \& Rosenthal, O. (1966), Mulec. Pharmacol. 2, 187-191. - 21. van DER WAERden, L. \& NieverGELT, E. (1956), Tafeln zum Vergleich zweier Stichproben mittels $\chi$-Test und Zeichentest, Springer-Verlag, Berlin-HeidelbergNew York.
Dr. H. W. Leber

Medizinische Kliniken und Polikliniken der Justus Liebig-Universität 63 Gießen

Klinikstr. $32 \mathrm{~b}$ 